\title{
Probing the relationship between BTBD9 and MEIS1 in C. elegans and mouse
}

\author{
Shangru Lyu ${ }^{1}$ (D), Atbin Doroodchi ${ }^{3}$, Yi Sheng ${ }^{2}$, Mark P. DeAndrade ${ }^{1}$, Youfeng Yang ${ }^{3}$, Yuning Liu ${ }^{1}$ (D), \\ Michael A. Miller ${ }^{3}$, Rui Xiao ${ }^{2}$ and Yuqing $\mathrm{Li}^{1, *}$ \\ ${ }^{1}$ Norman Fixel Institute for Neurological Diseases, Department of Neurology, College of Medicine, University of Florida, \\ Gainesville, FL, 32610, USA, ${ }^{2}$ Department of Aging and Geriatric Research, College of Medicine, University of Florida, \\ Gainesville, FL, 32610, USA, and ${ }^{3}$ Department of Cell, Developmental and Integrative Biology, the University of Alabama at \\ Birmingham, Birmingham, AL, 35294, USA \\ *Corresponding author. E-mail: yuqing.li@neurology.ufl.edu
}

(Received 22 January 2020; Revised 06 February 2020; Accepted 06 February 2020).

\begin{abstract}
Restless legs syndrome (RLS) is a neurological disorder characterized by an urge to move and uncomfortable sensations. Genetic studies have identified polymorphisms in up to 19 risk loci, including MEIS1 and BTBD9. Rodents deficient in either homolog show RLS-like phenotypes. However, whether MEIS1 and BTBD9 interact in vivo is unclear. Here, with $C$. elegans, we observed that the hyperactive egg-laying behavior caused by loss of BTBD9 homolog was counteracted by knockdown of MEIS1 homolog. This was further investigated in mutant mice with Btbd9, Meis1, or both knocked out. The double knockout mice showed an earlier onset of the motor deficit in a wheel running test but did not have increased sensitivity to heat stimuli as observed in single knock outs. Meis1 protein level was not influenced by Btbd9 deficiency, and $B t b d 9$ transcription was not affected by Meis1 haploinsufficiency. Our results demonstrate that MEIS1 and BTBD9 do not regulate each other.
\end{abstract}

Keywords: restless legs syndrome; hpo-9; unc-62; Btbd9; Meis1

\section{Introduction}

RLS is characterized by a strong urge to move and uncomfortable sensations in lower limbs, which can be relieved by movements. Genome-wide association studies have implicated up to 19 risk loci for RLS, across which two of the candidate genes are MEIS1 and BTBD9 (Schormair et al., 2017).

Knockout (KO) animals of BTBD9 or MEIS1 homologs exhibit RLS-like phenotypes. For instance, homozygous $B t b d 9 \mathrm{KO}$ mice have motor restlessness, disrupted sleep, and altered sensory perception (DeAndrade et al., 2012). Loss of BTBD9 homolog in Drosophila melanogaster results in increased motor activity, decreased dopamine levels, and disrupted sleep (Freeman et al., 2012). Heterozygous Meis1 KO mice are hyperactive (Meneely et al., 2018). C. elegans with decreased MEIS1 homolog show increased expression of ferritin (Catoire et al., 2011). Therefore, both BTBD9 and MEIS1 may play a role in the development of RLS, yet whether and how the two genes interact is not known.

\footnotetext{
(C) The Author(s) 2020. This is an Open Access article, distributed under the terms of the Creative Commons Attribution licence http:// creativecommons.org/licenses/by/4.0/), which permits unrestricted re-use, distribution, and reproduction in any medium, provided the original work is properly cited.
} 


\section{Objective}

Our goal was to define the relationships between BTBD9 and MEIS1 in the pathogenesis of RLS. Egg retention assay in C. elegans was used to determine if there are genetic interactions between $h p o-9$, a $B T B D 9$ homolog, and unc-62, a MEIS1 homolog. Furthermore, we created mouse models by knocking out BTBD9 homolog, Btbd9, MEIS1 homolog, Meis1, or both. Their motor-sensory responses were compared by wheel-running and tail-flick tests. Homozygous Meis1 KO was not included because of embryonic lethality (Spieler et al., 2014). The transcription of Btbd9 in Meis1 KO and the level of Meis1 protein in Btbd9 $\mathrm{KO}$ animals were studied.

\section{Methods}

C. elegans were maintained using standard methods (Catoire et al., 2011). The wildtype (WT) used was Bristol N2. The hpo-9 KO, hpo-9(tm3719), was obtained from the National BioResource Project (Japan) and backcrossed four times to the N2 background. RNAi against unc-62 (unc-62 RNAi) and the empty vector (EV) were used according to a standard feeding method with HT115 bacterial strain. Egg retention assay was performed according to published protocols (Chase \& Koelle, 2004) and analyzed by a Students' t-test (supplementary material).

Heterozygous Btbd9 KO (Lyu et al., 2019) were bred with Meis1 KO animals (Meneely et al., 2018) to generate double heterozygotes, which were bred with heterozygous Btbd9 $\mathrm{KO}$ mice to generate experimental mice. Behavioral tests were conducted as described (Lyu et al., 2019) and analyzed by SAS GENMOD or mixed model ANOVA. Western blot and quantitative RT-PCR were performed and analyzed as described (Yokoi et al., 2015) using striatal tissues (supplementary material).

\section{Results}

Worms: Figure 1 shows that unc-62 knockdown led to an increased number of eggs retained in both N2 and hpo-9(tm3719) as described (Kamath et al., 2003). The hpo-9 mutation caused fewer eggs retained in the presence or absence of unc-62 RNAi. Additionally, hpo-9(tm3719) treated with unc-62 RNAi retained a similar number of eggs as $\mathrm{N} 2$.

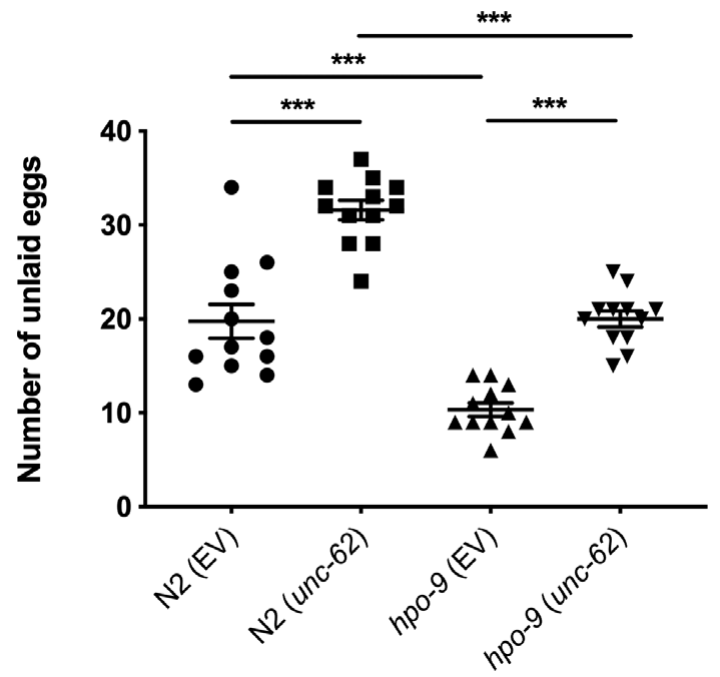

Figure 1. Egg retention assay. Bars represent the mean \pm standard error of the mean (SEM) for 12 animals for each strain. $\star \star \star, p<0.001$. 


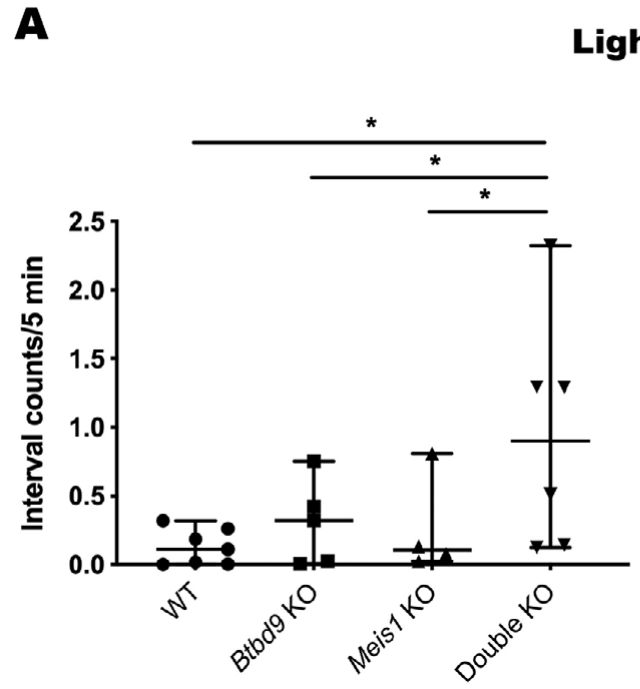

\section{Light phase}

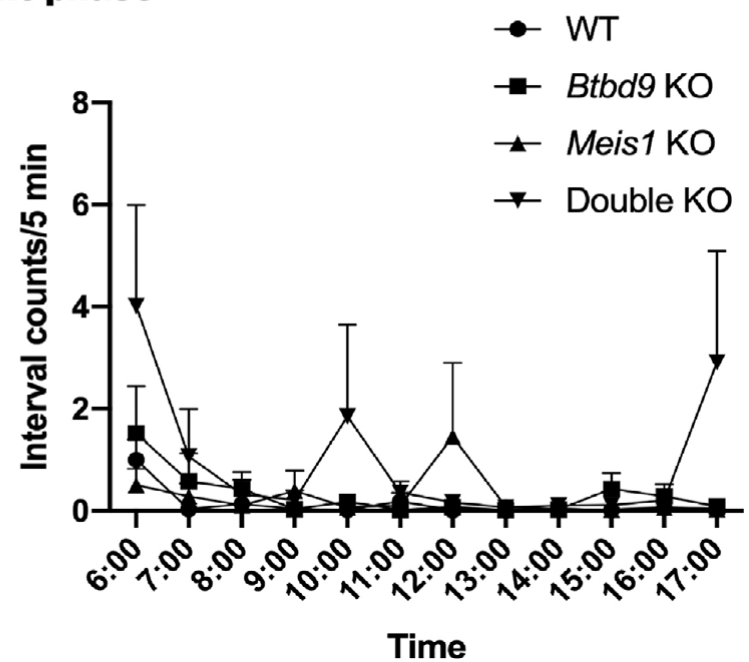

B

\section{Dark phase}
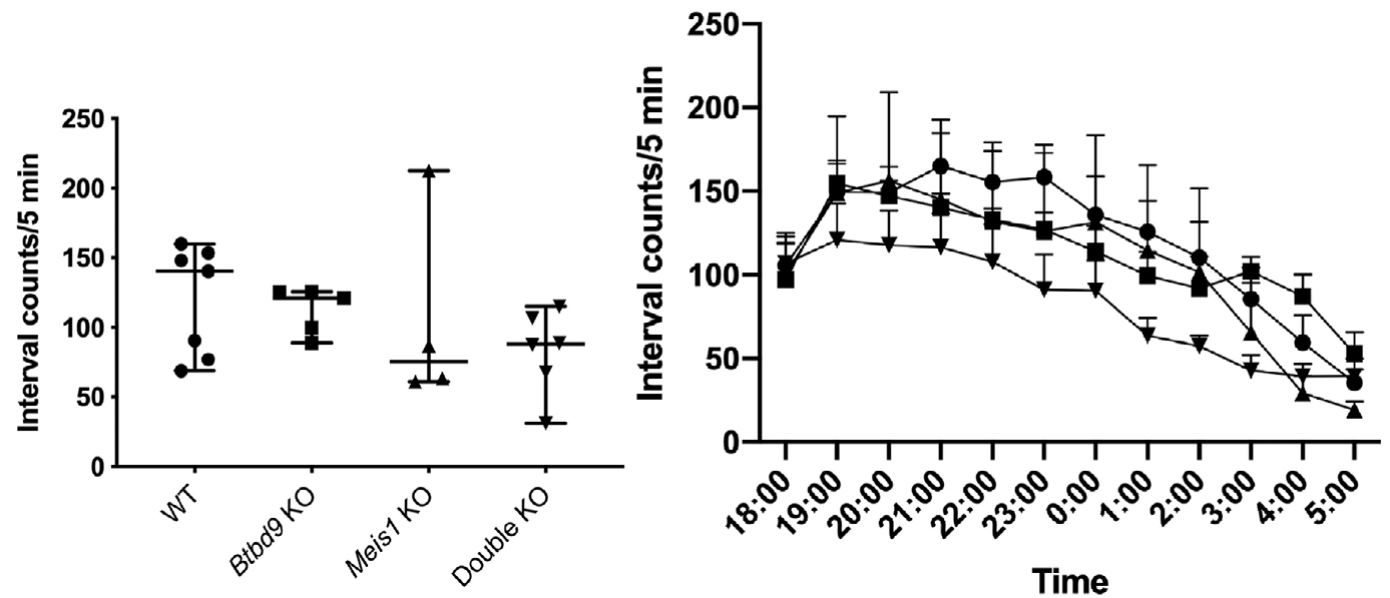

Figure 2. Wheel running during the light phase (A), and the dark phase (B). The data was not normally distributed and analyzed by SAS GENMOD with a negative binomial distribution. In the scatter plot, each dot is an average value calculated from 4 days' data for each mouse. Bars represent the median with $95 \%$ confidence intervals (Cls). Hourly activity is presented next to the scatter plot. Each dot is an average value calculated from 4 days' data for each genotype. The activity of the double KO mice shot up right after the light was turned on and right before the light was turned off. In addition, they also showed high levels of activity around the middle of the rest period. The results indicate that the double $\mathrm{KO}$ mice may have difficulty in falling asleep and tend to wake up early. WT, $\mathrm{n}=7$; Btbd9 KO, $\mathrm{n}=5$; Meis1 KO, $\mathrm{n}=4$; double $\mathrm{KO}, \mathrm{n}=6 .{ }^{*}, p<0.05$.

Mice: During the light phase of the wheel running test, neither single KOs exhibited significant difference compared with the WT (Figure 2). However, the double KO showed a robust increase from the WT and a lesser extent of increase from both single KOs. During the dark phase, activity levels were similar among the four groups. Figure 3 shows that the double KO did not have changes in the tail-flick response although both single KOs had reduced latency. Moreover, Meis1 protein levels and Btbd9 mRNA levels were unaffected by Btbd9 knockout and Meis1 deficiency, respectively (Figure 4). 


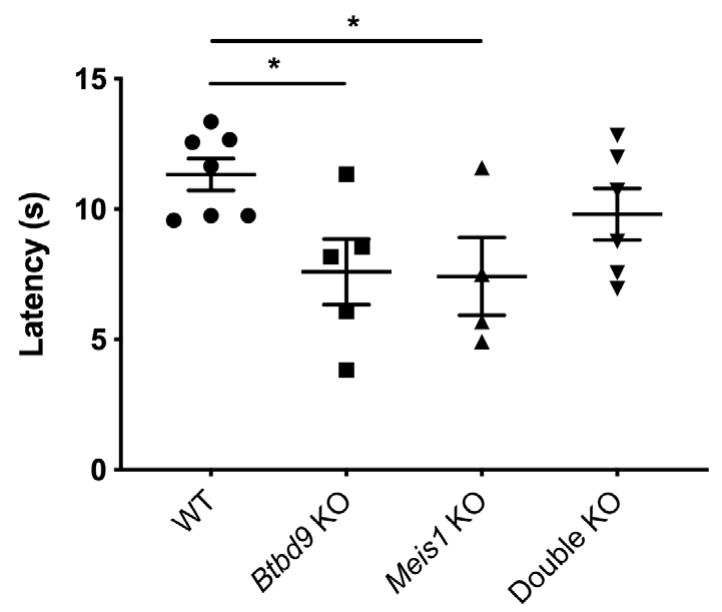

Figure 3. Tail-flick test. The data were normally distributed and analyzed by mixed model ANOVA with repeated measurements. Each dot is an average value calculated from 3 trials for each mouse. Single KO had reduced latency compared with the WT but did not show a significant difference compared with the double KOs. The double KO did not have significant changes compared with the WT. Bars represent the mean \pm SEM. WT, $n=7$; Btbd9 KO, $\mathrm{n}=5$; Meis1 KO, $\mathrm{n}=4$; double KO, $\mathrm{n}=6$. ${ }^{\star}, p<0.05$.

\section{Discussion}

Wheel-running data from day and night were analyzed separately because RLS symptoms mostly happen at night, which is the day for rodents. With animals at an average age of 3 months, we did not observe increased activity in either single KO as suggested before (DeAndrade et al., 2012; Meneely et al., 2018), indicating that the double $\mathrm{KO}$ had early-onset deficit while the single KOs were still asymptomatic. It has been shown that Btbd9 expression does not change by Meis1 deficiency (Spieler et al., 2014). This was confirmed by molecular analyses, suggesting that Btbd9 and Meis1 do not regulate each other.

\section{Conclusion}

In worms, the augmentation effect of unc-62 knockdown is independent of $h p o-9$ manipulation and it is also true the other way around. Moreover, $h p o-9$ knockout and unc-62 knockdown counteract each other. In mice, the wheel running test suggests that there is an additive effect of Meis 1 and Btbd9 mutations in the double KO mice. Btbd9 does not influence the Meis1 protein level, and Meis1 cannot alter Btbd9 gene expression. Hence, the two RLS risk genes work independently and have functional interactions in both worms and mice. Protein-protein interaction assays would be ideal to confirm this conclusion in the future.

Acknowledgments. We would like to thank Drs. Neil Copeland and Hesham Sadek for supplying Meis1 loxP mice, Dr. Shohei Mitani for the hpo-9(tm3719) strain, and Fumiaki Yokoi, Lin Zhang, Chad C. Cheetham, Sung Min Han, Jack Vibbert, Pauline Cottee, Jessica Winek, and Hieu Hoang for their technical assistance and stimulating discussions.

Author Contributions. SL, MPD, MAM, RX and YLi conceived and designed the study. SL, AD, YS, MPD, YY and YLiu conducted data gathering. SL and YLiu performed statistical analyses. SL and YLi wrote the article.

Funding Information. This work was supported by a grant from Restless Legs Syndrome Foundation; startup funds from the Departments of Neurology at UAB and UF; and the National Institutes of Health (grant numbers NS37409, NS47466, NS47692, NS54246, NS57098, NS65273, NS72872, NS74423, and NS82244). The content is solely the responsibility of the authors and does not necessarily represent the official views of the National Institutes of Health.

Publishing Ethics. The authors assert that all procedures contributing to this work comply with the ethical standards of the relevant national and institutional guides on the care and use of laboratory animals.

Conflicts of Interest. All authors declare none. 

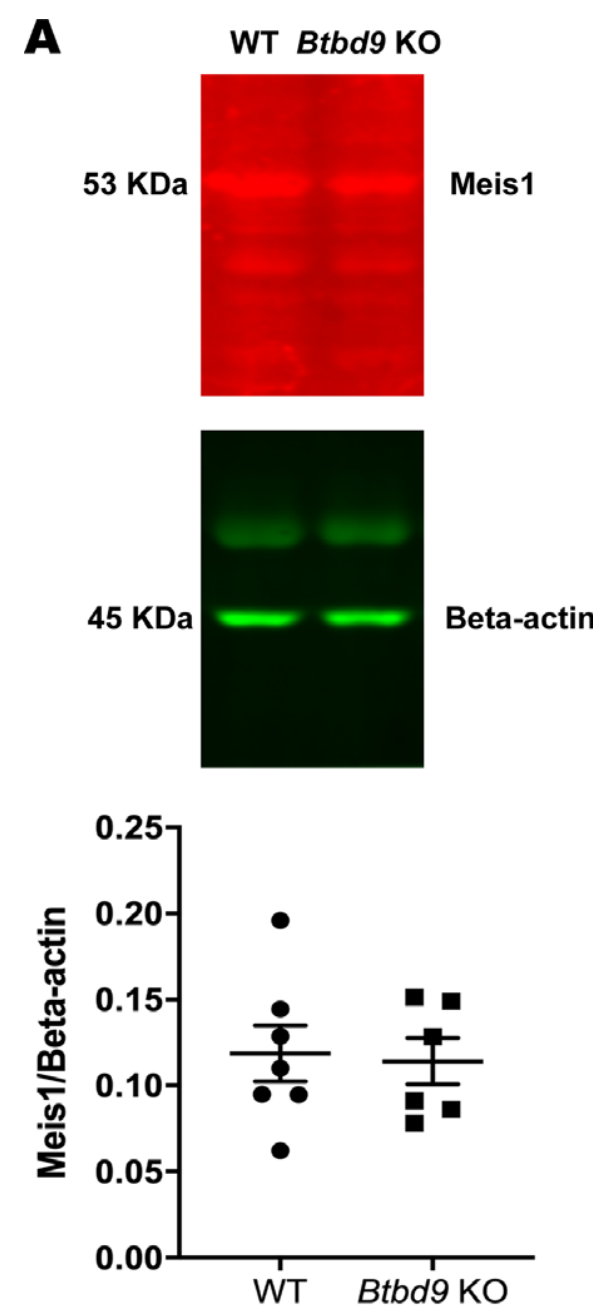

B

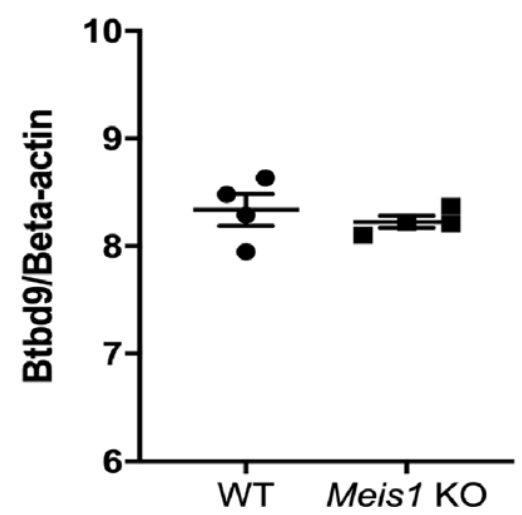

Figure 4. Molecular analysis. (A) Western blot to measure the amount of Meis1, normalized to $\beta$-actin, in $B$ tbd9 $\mathrm{KO}(\mathrm{n}=6)$ and WT $(n=7)$ mice. (B) Quantitative RT-PCR to test the level of Btbd9 mRNA, normalized to $\beta$-actin, in Meis1 KO $(n=4)$ and WT $(n=$ 4) mice. Bars represent the mean \pm SEM. 
Data Availability Statements. The data that support the findings of this study are available from the corresponding author upon reasonable request.

Supplementary Materials. To view supplementary material for this article, please visit http://dx.doi.org/10.1017/exp.2020.12.

\section{References}

Catoire, H., Dion, P. A., Xiong, L., Amari, M., Gaudet, R., Girard, S. L., Noreau, A., Gaspar, C., Turecki, G., Montplaisir, J. Y., Parker, J. A., \& Rouleau, G. A. (2011). Restless legs syndrome-associated MEIS1 risk variant influences iron homeostasis. Annals of Neurology, 70, 170-175.

Chase, D. L., \& Koelle, M. R. (2004). Genetic analysis of RGS protein function in Caenorhabditis elegans. Methods in Enzymology, 389, 305-320.

DeAndrade, M. P., Johnson, R. L., Jr., Unger, E. L., Zhang, L., van Groen, T., Gamble, K. L., \& Li, Y. (2012). Motor restlessness, sleep disturbances, thermal sensory alterations and elevated serum iron levels in Btbd9 mutant mice. Human Molecular Genetics, 21, 3984-3992.

Freeman, A., Pranski, E., Miller, R. D., Radmard, S., Bernhard, D., Jinnah, H. A., Betarbet, R., Rye, D. B., \& Sanyal, S. (2012). Sleep fragmentation and motor restlessness in a Drosophila model of restless legs syndrome. Current Biology, 22, 1142-1148

Kamath, R. S., Fraser, A. G., Dong, Y., Poulin, G., Durbin, R., Gotta, M., Kanapin, A., Le Bot, N., Moreno, S., Sohrmann, M., Welchman, D. P., Zipperlen, P., \& Ahringer, J. (2003). Systematic functional analysis of the Caenorhabditis elegans genome using RNAi. Nature, 421, 231-237.

Lyu, S., Xing, H., DeAndrade, M. P., Liu, Y., Perez, P. D., Yokoi, F., Febo, M., Walters, A. S., \& Li, Y. (2019). The role of BTBD9 in striatum and restless legs syndrome. eNeuro, 6, 0277-19.2019.

Meneely, S., Dinkins, M. L., Kassai, M., Lyu, S., Liu, Y., Lin, C. T., Brewer, K., Li, Y., \& Clemens, S. (2018). Differential dopamine D1 and D3 receptor modulation and expression in the spinal cord of two mouse models of restless legs syndrome. Frontiers in Behavioral Neuroscience, 12, 199.

Schormair, B., Zhao, C., Bell, S., Tilch, E., Salminen, A. V., Putz, B., Dauvilliers, Y., Stefani, A., Högl, B., Poewe, W., Kemlink, D., Sonka, K., Bachmann, C. G., Paulus, W., Trenkwalder, C., Oertel, W. H., Hornyak, M., Teder-Laving, M., Metspalu, A., \& Winkelmann, J. (2017). Identification of novel risk loci for restless legs syndrome in genome-wide association studies in individuals of European ancestry: A meta-analysis. The Lancet Neurology, 16, 898-907.

Spieler, D., Kaffe, M., Knauf, F., Bessa, J., Tena, J. J., Giesert, F., Schormair, B., Tilch, E., Lee, H., Horsch, M., Czamara, D., Karbalai, N., von, Toerne, C., Waldenberger, M., Gieger, C., Lichtner, P., Claussnitzer, M., Naumann, R., MüllerMyhsok, B., Winkelmann, J. (2014). Restless legs syndrome-associated intronic common variant in MEIS1 alters enhancer function in the developing telencephalon. Genome Research, 24, 592-603.

Yokoi, F., Dang, M. T., Liu, J., Gandre, J. R., Kwon, K., Yuen, R., \& Li, Y. (2015). Decreased dopamine receptor 1 activity and impaired motor-skill transfer in Dyt1 DeltaGAG heterozygous knock-in mice. Behavioural Brain Research, 279, $202-210$.

Cite this article: Lyu S, Doroodchi A, Sheng Y, DeAndrade MP, Yang Y, Liu Y, Miller MA, Xiao R, Li Y (2020). Probing the relationship between BTBD9 and MEIS1 in C. elegans and mouse Experimental Results, 1, e8, 1-10. https://doi.org/10.1017/ exp.2020.12 


\section{Peer Reviews}

\section{Reviewing editor: Dr. Michael Nevels}

University of St Andrews, Biomolecular Sciences Building, Fife, United Kingdom of Great Britain and Northern Ireland, KY16 9ST

This article has been accepted because it is deemed to be scientifically sound, has the correct controls, has appropriate methodology and is statistically valid, and met required revisions.

doi:10.1017/exp.2020.12.pr1

Review 1: Probe the relationship between BTBD9 and MEIS1 in C. elegans and mouse

Reviewer: Dr. Aaro Salminen (iD

Helmholtz Zentrum München, Institute of Neurogenomics, Neuherberg, Germany

Date of review: 05 February 2020

Published online:

(C) The Author(s) 2020. This is an Open Access article, distributed under the terms of the Creative Commons Attribution licence http://creativecommons.org/licenses/by/4.0/), which permits unrestricted re-use, distribution, and reproduction in any medium, provided the original work is properly cited.

Conflict of interest statement. Reviewer declares none

\section{Comment}

Comments to the Author: The manuscript details mouse and worm experiments on BTBD9 and MEIS1, two major candidate genes of RLS. As RLS is a polygenic disease, the double knock-out experiment is of great value in determining the relationship as well as a possible additive effect of these genes to the susceptibility to RLS. However, there are concerns relating to the analysis of the data which should be addressed.

In Figures 2 and 3, individual measurements resulting from repeated measurements in the same animal should not be displayed as individual data points, but should be first averaged within animal.

In statistical tests relating to Figs 2-3, was repeated measures ANOVA used? The test used should be stated in the legend. If so, the appropriate way to analyse the data would be to average first within animal and then use for example one-way ANOVA.

Division into light and dark period is used for wheel running activity, as is often done for this kind of data. Rather, the period right before and after the lights-on should be looked at. This would correspond better to human RLS and allow comparison with previous data in MEIS1 knock-out mice.

In the introduction, the sentence describing the human genetics of RLS is somewhat confusing and should be reformulated.

What does " 4 repeats for each genotype" mean? Does this mean the last 4 days that were used in the analysis according to Lyu et al. 2019 or did the mice spend four times seven days in the running wheel?

\section{Score Card}

Presentation

Does the paper cite relevant and related articles appropriately? (30\%) 
Does the abstract correctly embody the content of the article? (25\%)

Does the introduction give appropriate context? (25\%)

Is the objective of the experiment clearly defined? (25\%)

Analysis

Does the discussion adequately interpret the results presented? (40\%)

Is the conclusion consistent with the results and discussion? (40\%)

Are the limitations of the experiment as well as the contributions of the experiment clearly outlined? (20\%) 


\section{Review 2: Probe the relationship between BTBD9 and MEIS1 in C. elegans and mouse}

Reviewer: Dr. Guy A. Rouleau (ID

McGill University, Neurology and Neurosurgery, Montreal, Canada, H3A 0G4

Date of review: 05 February 2020

Published online:

(C) The Author(s) 2020. This is an Open Access article, distributed under the terms of the Creative Commons Attribution licence http://creativecommons.org/licenses/by/4.0/), which permits unrestricted re-use, distribution, and reproduction in any medium, provided the original work is properly cited.

Conflict of interest statement. Reviewer declares none.

\section{Comment}

Comments to the Author: In this paper Lyu et al. investigated the impact of two RLS candidate genes in the sensory-motor characteristics of C. elegans and mice. They also probed the potential interactions of these two genes based on the sensory-motor effects of their double KOs in the animals. This is an interesting paper that can provide useful information on the roles of MEIS1 and BTBD9 as two significant genes in RLS genetics.

The paper is well written, and the experiments are well conducted. The Western blot analysis results are not included in the supplemental data, and only graphs with quantitative measurement of the protein levels are provided.

Minor revisions:

1. Lines 39-41 "The double knockout mice showed an earlier onset of the motor deficit in the wheel running test but did not have increased sensitivity to the heat stimuli as observed in single KOs." Based on publications by Salminen et al. 2017, Meneely et al. 2018 and Spieler et al. 2014, the sensitivity to heat stimuli was not observed in mice with Meis1 deficiency. The authors could provide references supporting the observation of increased sensitivity to heat stimuli in BOTH KOs.

2. Could the authors elaborate on why Meis1 was only measured at protein level (WB), but Btbd9 at mRNA level (q-RT-PCR)? Would have been ideal if both genes were measured at both levels. The reason and limitations should be discussed in the paper.

3. Lines 84-85 "With or without unc-62 RNAi, hpo-9(tm3719) retained fewer eggs than N2." According to Figure 1, "N2" should be "N2(unc-62)".

4. To make it easier for the readers, in the results section, it should be more clearly stated that which paragraph refers to which animal.

5. Line 87 "fo" is a typo?

6. Lines 90-91. The two sentences should be separated more clearly. The second sentence seems to continue the previous sentence about dark phase activity.

7. Line 122. "the two RLS risk genes work independently and have functional interactions in both worms and mice." The authors are suggesting that there are functional interactions between the two proteins. A protein-protein interaction assay would be ideal to confirm this interaction. If not feasible, the limitations of this conclusion should be discussed in the manuscript.

8. Please provide all the original Western blot figures in the supplemental data. 
Is the data presented in the most useful manner? (40\%) 4/5

Does the paper cite relevant and related articles appropriately? (30\%) 5/5

\section{Context}

Does the abstract correctly embody the content of the article? (25\%)

Does the introduction give appropriate context? (25\%)

Is the objective of the experiment clearly defined? (25\%)

Are the limitations of the experiment as well as the contributions of the experiment clearly outlined? (20\%) 